\title{
Die Kommissionsvorschläge zur alternativen und Online-Streitbeilegung: Ist der Optimismus gerechtfertigt?
}

\author{
Jim Davies
}

(C) VOe 2012

Der europäische Verbraucher hat in Hinblick auf Wichtigkeit und Status ein neues Niveau erreicht: Denn er genießt mittlerweile Anerkennung als immaterieller Kapitalstock. Nach einhelliger Ansicht werden die neuen mächtigen Verbraucher Wettbewerb und Innovation ankurbeln, Unternehmen zur Lieferung qualitativ hochwertiger Güter zwingen sowie Wirtschaftswachstum und Nachhaltigkeit stark beeinflussen. ${ }^{1}$ Um diesen immateriellen Kapitalstock noch weiter zu motivieren, wurden jüngst zwei Vorschläge zur alternativen Streitbeilegung (AS) und Online-Streitbeilegung (OS $)^{2}$ vorgestellt, die den außergerichtlichen Rechtsschutz für Verbraucher verbessern sollen. Die Begründungen und Erläuterungen zu den neuen Vorschlägen klingen überwältigend positiv. Vor allem die Folgenabschätzung der Kommission erwartet, dass bessere Information über und vermehrter Einsatz von AS die Verbraucher motivieren wird, vermehrt Waren und Dienstleistungen online und grenzüberschreitend zu erwerben. ${ }^{3}$

\footnotetext{
${ }^{1}$ Special Eurobarometer 342, Consumer Empowerment 5 and 6, abrufbar unter: <ec.europa.eu/consumers/consumer_empowerment/docs/ report_eurobarometer_342_en.pdf>.

${ }^{2}$ Vorschlag für eine Richtlinie des Europäischen Parlaments und des Rates über Formen der alternativen Beilegung verbraucherrechtlicher Streitigkeiten und zur Änderung der Verordnung (EG) Nr. 2006/2004 und der Richtlinie 2009/22/EG (Richtlinie über alternative Streitbeilegung) COM (2011) 793 final und Vorschlag für eine Verordnung des Europäischen Parlaments und des Rates über die Online-Beilegung verbraucherrechtlicher Streitigkeiten (Verordnung über Online-Streitbeilegung) COM (2011) 794 final.

${ }^{3}$ SEC (2011) 1408 final 66 (nicht in deutscher Sprache verfügbar).
}

Dr. Jim Davies $(\varangle)$ University of Northampton, Park Campus, Boughton Green Road, Northampton NN2 7AL, United Kingdom E-mail: <jim.davies@northampton.ac.uk>; übersetzt von Kristin Nemeth
Nach der offiziellen Folgenabschätzung werden die neuen Maßnahmen ,positive soziale Auswirkungen” haben, weil sie allen Verbrauchern, somit auch den besonders verletzlichen, erlauben werden, ihre Streitigkeiten vorzutragen und einen Ausgleich für Einbußen zu erhalten. Da diese Ausgleichszahlungen somit über den Verbraucher wieder dem Binnenmarkt zufließen, werden sie unweigerlich zum Wirtschaftswachstum beitragen. Gleichzeitig wird die OSPlattform die Bereitschaft der Unternehmen, grenzüberschreitend anzubieten, erhöhen. Unternehmen werden eher bereit sein, im elektronischen Geschäftsverkehr aktiv zu sein, Verbraucher werden Zugang zu mehr Produkten und Dienstleistungen erlangen, am Markt wird mehr Wettbewerb entstehen und die Preise werden fallen. Die Folgenabschätzung geht sogar so weit zu sagen, dass sich durch die verstärkte Nutzung von AS und OS eine positive Eigendynamik entwickeln wird: Die den neuen Maßnahmen zurückhaltend gegenüberstehenden Unternehmen automatisch ausgeschlossen werden und diese den Verlust ihres Ansehens befürchten müssen, während umgekehrt die ASfreundlichen Unternehmen ihr gutes Ansehen bewahren und mit ihren Kunden gute Geschäftsbeziehungen aufbauen werden, und zwar ,online wie offline, bei Binnen- und bei grenzüberschreitenden Geschäften, bei Waren und bei Dienstleistungen“. 4

Die beschriebene Eigendynamik (kostengünstige Streitschlichtung, verbesserte Unternehmensansehen, erhöhte Produkt- und Dienstleistungsgüte und geringere Nachteile für Verbraucher) skizziert ein Marktmodell, in dem Verbraucher und Unternehmer ihre Eigeninteressen auf faire Art und Weise verfolgen. Der Folgenabschätzung zufolge haben die Verbraucher in diesem Modell die Möglichkeit ,,alle ihre Streitigkeiten in einem AS-Verfahren zu verhandeln ... un-

\footnotetext{
${ }^{4}$ SEC (2011) 1408 final 67.
} 
abhängig vom betroffenen Geschäftszweig und unabhängig vom Land, wo die Ware gekauft oder die Dienstleistung erbracht wurde" . ${ }^{5}$ Vielleicht sind wir von diesem wirtschaftlichen Nirvana tatsächlich nur eine Richtlinie und eine Verordnung weit entfernt; ein wenig Zynismus erscheint mir an dieser Stelle allerdings angebracht..

Verbraucherrechtsschutz ist ein komplexes Thema; die Wirksamkeit der Maßnahmen hängt hier ua auch vom Verhalten des Verbrauchers ab, das wiederum von kulturellen Gegebenheiten, individuellen Fähigkeiten und Vertrauen, sowie von sozialdemographischen Komponenten, den Kräften im Markt und schließlich auch von gesetzgeberischen Maßnahmen beeinflusst ist. Das Europäische Parlament hat mit gutem Grund davor gewarnt, dass zu viele verschiedene AS-Systeme den Verbraucher verwirren und zu gemischten Resultaten führen können: Schon heute gibt es über 750 europäische AS-Institutionen, die freilich im Verbraucher- und Unternehmerbewusstsein wenig verankert und von zT großen Unterschieden geprägt sind. Angesiedelt sind sie entweder bei der öffentlichen Hand oder dem entsprechenden Industriezweig finanziert werden sie privat oder öffentlich oder in Kombination von beidem; räumlich decken sie zT das gesamte Staatsgebiet ab, zT aber auch nur eine Region oder eine Gemeinde; teils widmen sie sich nur einem bestimmten Geschäftszweig, teils bedienen sie eine ganze Branche oder sogar mehrere Branchen; Entscheidungen werden von Gremien oder von Einzelpersonen getroffen; sie können bindend sein oder lediglich Empfehlungen darstellen. ${ }^{6}$

Greift man als Beispiel den Energiesektor heraus, so zeigt sich, dass das Dritte Energiepaket die Mitgliedstaaten zwar zur Einführung von AS verpflichtet hat, ${ }^{7}$ dass dies aber nicht lückenlos erfolgte; ebenso hinken das Verbraucherund das Unternehmerbewusstsein der Entwicklung hinterher und wurden die wichtigsten - in zwei Empfehlungen der Kommission ${ }^{8}$ enthaltenen - Prinzipien nicht befolgt. ${ }^{9}$ Bei der Umsetzung solch sektoraler Maßnahmen zeigen sich somit bereits Defizite. Darüber hinaus besteht Bedarf an $z u$ sätzlichen Maßnahmen, um besonders verletzliche Verbraucher zu schützen; eine Unterscheidung, die in den neuen Vorschlägen zu AS und OS übrigens ebenso nicht vorkommt.

\footnotetext{
${ }^{5}$ SEC (2011) 1408 final 65.

${ }^{6}$ Siehe SEC (2011) 1408 final 10 und 16.

${ }^{7}$ Art 3 Abs 13 RL 2009/72/EG und Art 3 Abs 9 RL 2009/73/EG.

${ }^{8}$ Working Group Report on Alternative Dispute Resolution in the Energy Sector 2, abrufbar unter: <ec.europa.eu/consumers/citizen/ my_rights/docs/energy_adr_report_en.pdf>.

${ }^{9}$ Vgl die Empfehlungen der Kommission 98/257/EG 31 und 2001/310/ EG 56.
}

Auch bestimmte Verbraucherverhaltensweisen können den Optimismus rund um die beiden neuen Vorschläge schmälern, und zwar die Vorliebe, persönlich einzukaufen, ob aus Treue bestimmten Geschäften gegenüber oder schlicht aus Gewohnheit. Rund 40\% der Verbraucherprobleme werden bereits zur Zufriedenheit aller Beteiligten zwischen Verbraucher und Unternehmer gelöst; in nur knapp einem Viertel der Fälle sind die beteiligten Verbraucher der Ansicht, dass die geringe Schadenshöhe den Aufwand einer Rechtsverfolgung nicht rechtfertige. Trotz Vorhandenseins von rund 750 AS-Institutionen wissen etwa $80 \%$ der Verbraucher nichts von deren Existenz; rund 25\% der Verbraucher geben an, dass sie AS-Maßnahmen nicht ergreifen würden, weil dies zu große Anstrengung, zu hohe Kosten und zu lange Verfahren mit sich brächte. ${ }^{10}$ Demographische Analysen zeigen eine erhebliche Abweichung im Verbraucherverhalten, sowohl zwischen den einzelnen Mitgliedstaaten (kulturelles Verbraucherverhalten) als auch innerhalb der Mitgliedstaaten (sozialwirtschaftlicher Status). Die verletzlichsten Verbraucher sind die Alten, die schlecht Ausgebildeten und speziell solche, die noch nie einen Computer benutzt haben und auch das Internet nicht nützen; bei dieser Gruppe handelt es sich freilich gleichzeitig um die Verbraucher, die am ehesten einen Schaden erleiden und die angeben, sicher keine AS in Anspruch nehmen zu wollen. ${ }^{11}$

Bei ihrem Versuch, eine Pauschallösung für mehr Verbraucherwohlbefinden $\mathrm{zu}$ finden, gehen die neuen Vorschläge von einem etwas idealisierten und beschönigten Modell des Verbraucherrechtsschutzes aus. Die existierenden AS-Regime sind zu unterschiedlich, zT eingefahren und zu lückenhaft, um eine Basis für ein einheitliches Schema europaweit oder auf Mietgliedstaatenebene darzustellen. Das Verbraucherverhalten zeigt, dass der rhetorische Optimismus rund um die Vorschläge übertrieben ist. Unzweifelhaft kann AS sowohl für den Verbraucher als auch für Unternehmer und Markt von erheblichem Nutzen sein. ${ }^{12}$ Fraglich ist jedoch, ob diese neuen Vorschläge das Potential haben, als Wegbereiter einer positiven Eigendynamik zu wirken, bei welcher das Gemeinwohl durch eigeninteressiertes Handeln von Verbraucher und Unternehmer gefördert wird.

\footnotetext{
${ }^{10}$ Special Eurobarometer 342 (Fn 1) 210.

${ }^{11}$ Eurobarometer 342 (Fn 1) 225.

${ }^{12}$ SEC (2011) 1408 final 7, 172.
} 